\title{
Experience and Results from the 6 Megapixel PILATUS System
}

\author{
Anna Bergamaschi, Christian Broennimann, Eric Eikenberry, Beat Henrich', \\ Miroslav Kobas, Philipp Kraft, Bernd Schmitt \\ Paul Scherrer Institut \\ 5232 Villigen PSI, Switzerland
}

PILATUS detector systems operate in single photon counting mode and are based on the newly developed hybrid-pixel technology [8], [9], [10] and [11]. The main difference to existing detectors is that $\mathrm{X}$-rays are directly transformed into electric charge and processed in the CMOS readout chips. This design shows no leakage current or readout noise and leads to a high dynamic range of 1,000,000 (20 bits) per pixel, short readout times of less than $3.5 \mathrm{~ms}$, a high framing rate of over 200 images per second and an excellent point-spread function. The quantum efficiency of the $0.32 \mathrm{~mm}$ thick silicon sensor is optimal for experiments in the energy range from 3 to $15 \mathrm{keV}$, however the detectors can be used for energies of up to $40 \mathrm{keV}$. Using the paralyzable counter model a dead time of $200.4 \mathrm{~ns}$ (standard gain settings) was measured [7]. This leads to a counting rate of almost $2 \times 10^{6}$ photons/pixels/s, well suited to handle the high flux of modern synchrotron light sources.

Keywords: Hybrid pixel detector, Single Photon Counting, Macromolecular Crystallography

The 16th International Workshop on Vertex detectors

Lake Placid, NY, USA

23-28 September, 2007

\footnotetext{
$1 \quad$ Speaker
} 


\section{Introduction}

A PILATUS hybrid-pixel detector is composed of a silicon sensor, which is a twodimensional array of pn-diodes processed in high-resistivity silicon, connected to an array of readout channels designed with advanced CMOS technology (Fig. 1). Each readout channel is connected to its corresponding detecting element through a microscopic indium ball, with a typical diameter of $18 \mathrm{um}$. This process is called microbump-bonding and was refined at the Paul Scherrer Institut (PSI).

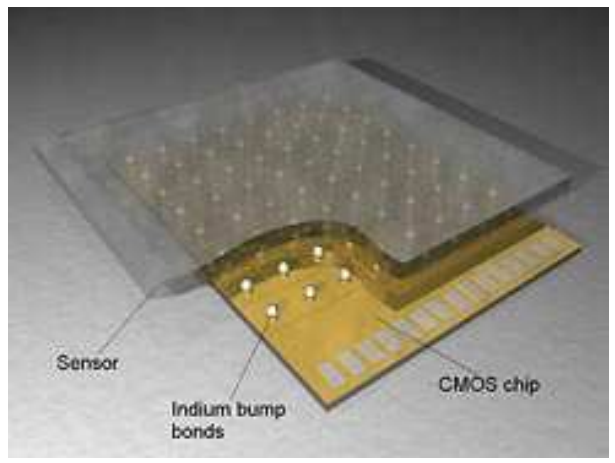

Figure 1: Hybridization technology by microbump-bonding.

The great advantage of this approach is that standard technologies are used for the silicon sensor and the CMOS readout chips [1], which guarantees highest quality. Both of them can be optimized separately, as the best silicon substrates for X-ray detection and for high-speed/highquality electronics are very different. Moreover, the small size of the pixel $\left(172 \times 172 \mu \mathrm{m}^{2}\right)$ and of the interconnection results in a very low capacitance, which has the beneficial effect of reducing noise and power consumption of the pixel readout electronics.
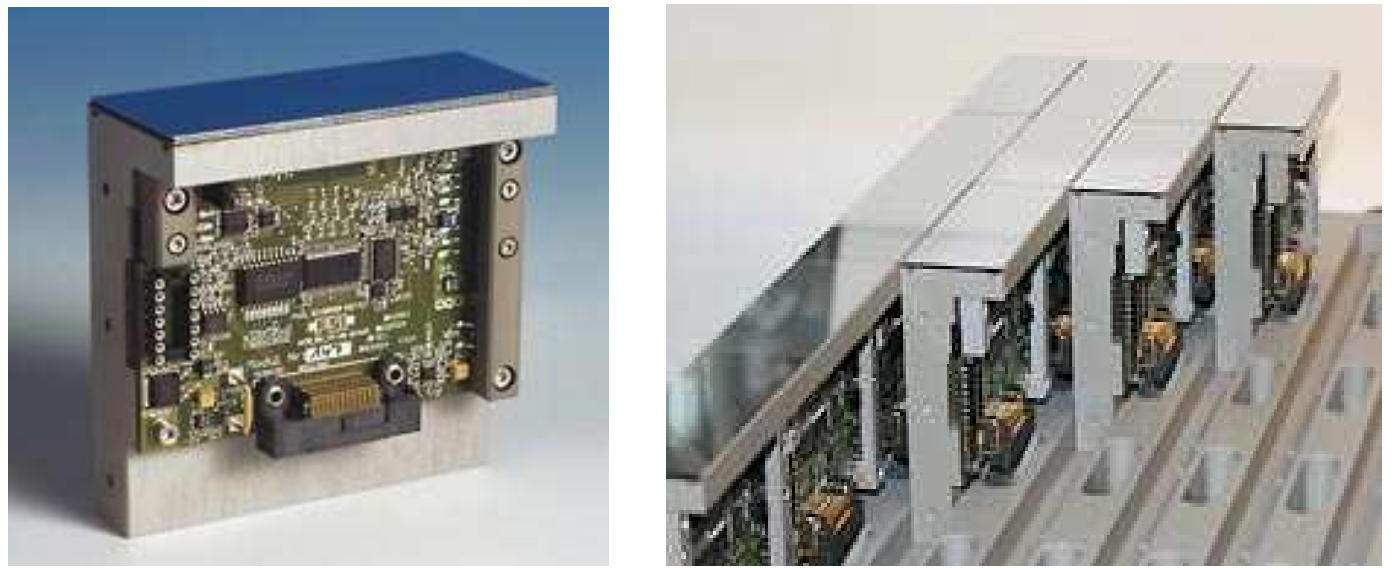

Figure 2: Single detector module (left). Modules assembled to multimodules (right). 
The fundamental unit of the PILATUS detectors, the module, consists of a single fully depleted monolithic silicon sensor with an $8 \times 2$ array of CMOS readout chips bump-bonded to it. To span the gaps between two adjacent chips two rows (columns) of larger pixels with a size of 3/2 of a normal pixel $(258 \mu \mathrm{m} \times 172 \mu \mathrm{m})$ are introduced. The counts of two of these pixels are distributed in software into 3 virtual pixels after readout. Thus each sensor is a continuous array of $487 \times 195=94^{\prime} 965$ pixels without dead areas and covers an active area of $83.8 \times 33.5$ $\mathrm{mm}^{2}$. This combined unit is wire-bonded to the mounting bracket with its readout electronics to form the module (Fig. 2, left). These modules are mounted to a high-precision mechanical frame to realize multimodules setups with up to 60 modules (Fig. 2, right).

\section{The PILATUS II CMOS Readout Chip}

The CMOS readout chip is designed and fabricated in the UMC1 $0.25 \mu \mathrm{m}$ technology using radiation tolerant design. The chip comprises an array of identical pixels containing a charge sensitive amplifier, a single level comparator, a counter and its control logic (Fig. 3). Every pixel is addressable according to its column and row number. To operate the pixels some additional logic is needed, the so called column control that is located in the periphery. Two main modes of operation are defined: in the counting mode all pixels are individually counting incoming X-rays; in the readout mode stored data is transferred to the output pad (DOUT).

PILATUS II Chip: 60 columns, 97 rows

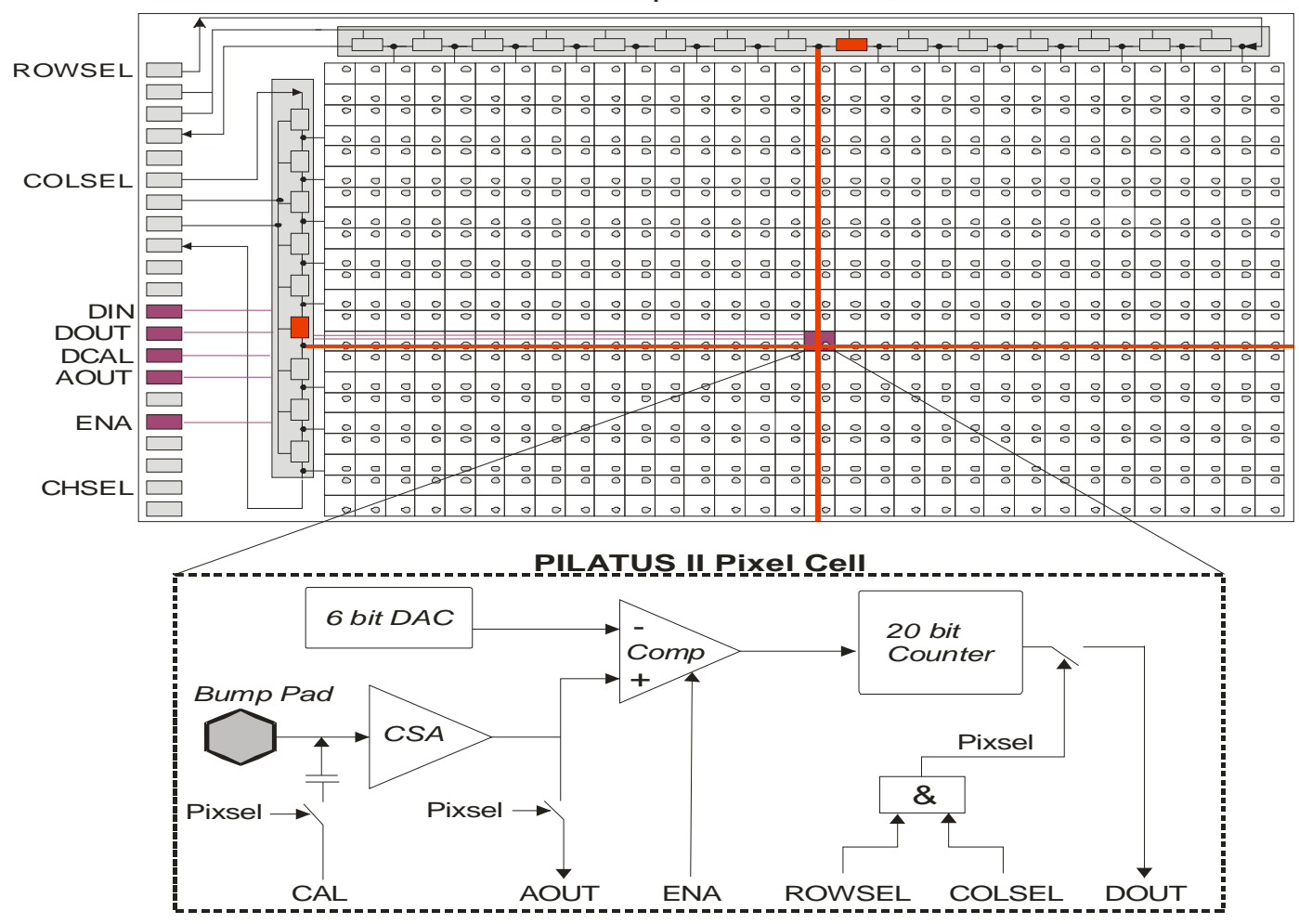

Figure 3: Schematic readout architecture of the PILATUS II chip and schematic close-up of a single pixel

\footnotetext{
${ }^{1}$ UMC: United Microelectronics Corporation, Taiwan.
} 
The analog part of the pixel contains a charge sensitive preamplifier and an AC-coupled shaper in series. The working principle of this circuit is based on a first amplification of the input signal coming from the sensor performed by the charge sensitive preamplifier. This charge is then differentiated and integrated by the capacitance of the shaper to decrease the noise content of the signal [2]. The voltage step on the output of the preamplifier creates a charge which is integrated in the shaper. To observe the amplifier output directly an arbitrary pixel can be multiplexed onto the analog out bus of the column and from there to the analog output (AOUT) of the chip.

A comparator is coupled to the shaper output. To reduce the threshold dispersion, each pixel has a 6 bit DAC for trimming. The digital part of the pixel consists of a clock generator, a 20 bit counter and its control logic. The output of the comparator stimulates the clock generator which increments the counter by one.

The chip-periphery contains an Input/Output part, a row selection shift register and column control logic with the column selection shift register. The chip needs to be addressed by setting the signal CHSEL to logic high state. This is necessary for the operation of a chip on a multi chip module. Each pixel of the chip can be addressed with the row and column shift register for programming, readout and diagnostics.

In counting mode (ENA is in logic high state), no pixel is selected and all of them count incoming X-rays in parallel. The readout mode is defined by selecting a pixel. The bit content of its counter is transferred to the output serially by an external clock signal. The full readout sequence of the chip is thus a sequential selection of each pixel and 20 clock pulses. In current operation designs with $50 \mathrm{MHz}$ clock speed full chip readout takes $3.5 \mathrm{~ms}$.

\section{The PILATUS Module}

The PILATUS module (Fig. 2) consists of a single, fully depleted monolithic silicon sensor with an $8 \times 2$ array of CMOS readout chips. The architecture is chosen such that all chips can be operated in parallel with the consequence that the single chip readout time of $3.5 \mathrm{~ms}$ can be preserved on module level. The fabrication steps require high precision positioning tools to place the modules on the mounting bracket. Here, a HDI (High Density Interconnect) distributes the logic- and power signals for each chip and provides the bond-pads for wire-bonding. A dedicated PCB-board delivering the different supply voltages (MCB or Module Control Board) is connected to the HDI.

\subsection{Single- and Multimodule Pilatus Detectors}

The main philosophy pursued, was driven by the intention to keep the massive parallel readout as long as possible. The data is finally serialized and send over high-speed link to the detector PC. On module level, the module control board (MCB) supports parallel readout of the 16 chips. In case of multi-module detectors several modules are grouped to a bank (Fig. 4). The Bank Control Board (BCB) allows parallel readout of the modules and temporarily stores the data until they are fetched by the Detector Control Board (DCB). On the BCB each module has a corresponding FPGA to control the data flow in combination with a FIFO where the module 
data is stored. Depending on the needs for speed, the DCB can be connected to only one or to a series of BCBs.

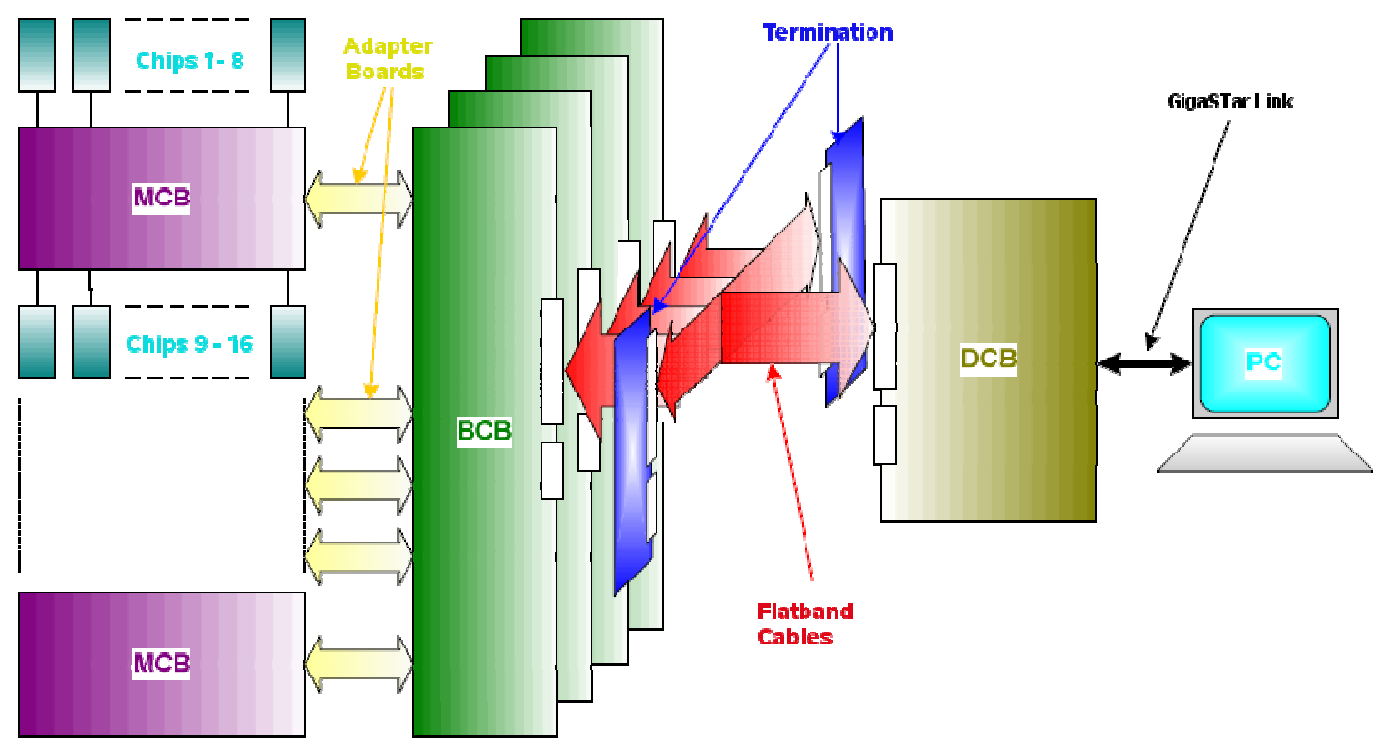

Figure 4: The multimodule read-out architecture

The key feature of the data transfer between detector and data acquisition PC is the GigaSTaR technology which is a universal high-speed point-to-point communication link. The nominal transfer load on this serial link is $1.3 \mathrm{Gbit} / \mathrm{s}$. Subtracting the protocol overhead we have a payload of $120 \mathrm{Mbytes} / \mathrm{s}$. On the PC side there is a PCI card holding the counterpart for the communication. The GigaSTaR high speed serial link chips in combination with a XILINX Virtex II FPGA are the main components of the DCB. The task of the FPGA is to control the communication and take care of the digital signals for the pixel chips. Although the main data flow is always in one direction (from detector to PC), both links, the upper parallel as well as the serial are duplex and therefore allow sending commands from the PC up to the DCB and from there further to the $\mathrm{BCB}$ or to the $\mathrm{MCB}$. The data arriving from the $\mathrm{BCB}$ or the $\mathrm{MCB}$ parallel (16 bits wide / $100 \mathrm{MHz}$ ) is then converted to a serial stream and sent over the GigaSTaR® link.

The PILATUS 100K detector achieves with full readout (all 20 bits/pixel) a frame rate of $200 \mathrm{~Hz}$. Exposure times can be varied from $100 \mathrm{~ns}$ to hours and are controlled either internally with a precision of $15 \mathrm{~ns}$ or by applying an external gate signal.

The PILATUS 6M system (Fig. 5, left) is a matrix of 5x12 modules. One DCB controls 6 BCBs. This architecture leads to a maximum frame rate of $12.5 \mathrm{~Hz}$ including data compression on the detector PC. Uncompressed, a single image has a size of 24 MBytes.

For the PILATUS $2 \mathrm{M}$ system (Fig. 5, right) a $3 \times 8$ modules matrix is controlled by two DCBs reading out $4 \mathrm{BCBs}$ each. Here, a maximum frame rate of $30 \mathrm{~Hz}$ is achieved. 

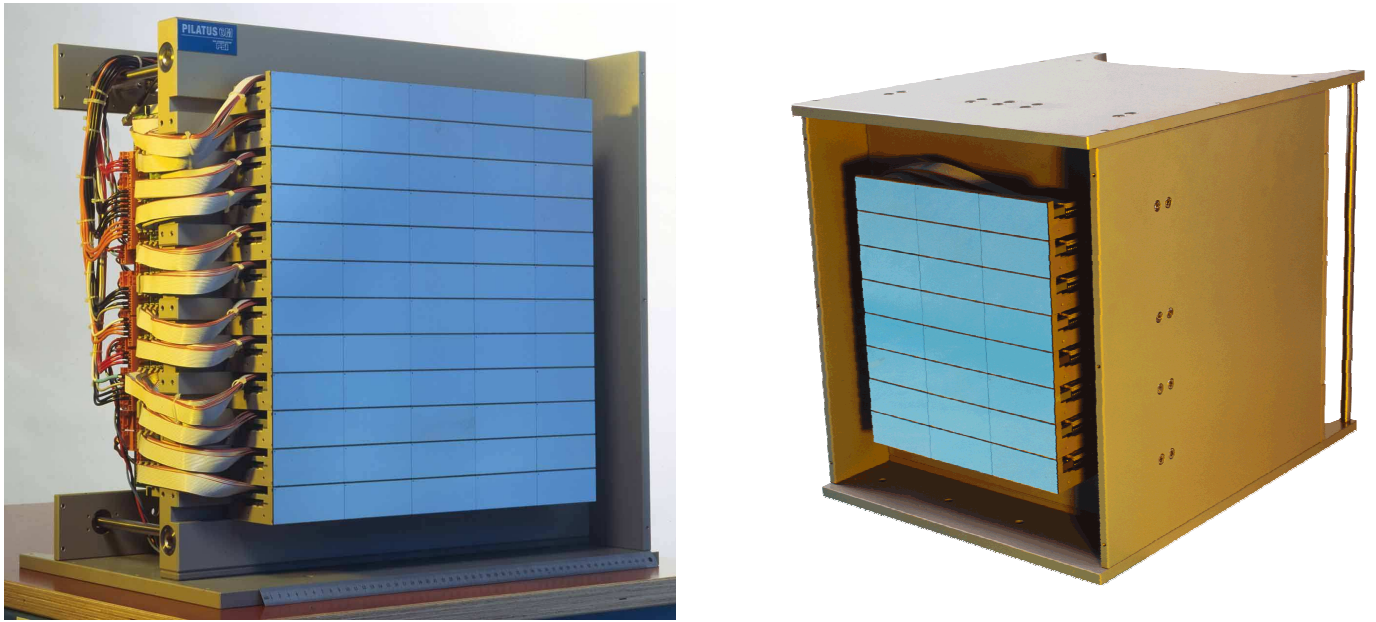

Figure 5: The different multimodule systems. Left PILATUS 6M (5x12 modules). Right: PILATUS 2M (3x8 modules).

\section{Detector Properties and its Applications}

Each of the systems described has its dedicated fields of application and benefit from various improvements compared to common CCD systems. In the following, a compact overview of the results from different experiments using PILATUS detectors will be given.

\subsection{Protein Crystallography}

The high dynamical range of the PILATUS pixel in combination with noise suppression due to the use of a threshold perfectly matches the requirements of protein crystallography.
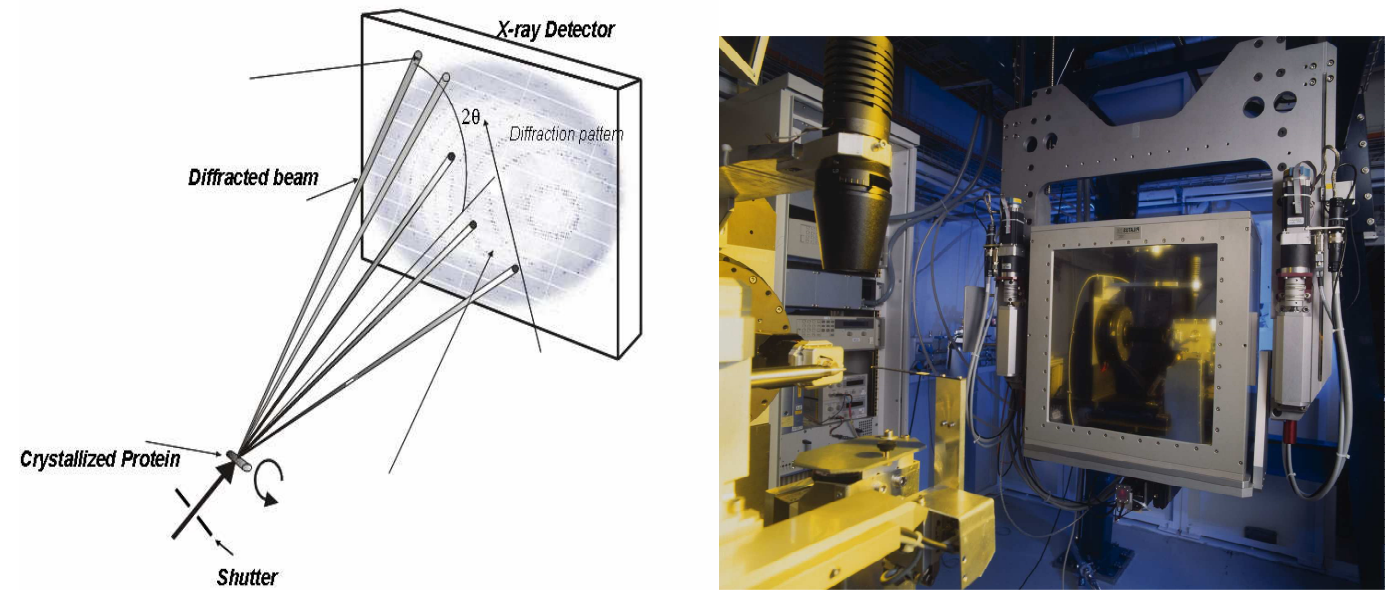

Figure 6: Typical Protein Crystal diffraction setup. Left: A focused X-ray beam hits a crystal which is rotated by a fraction of its mosaic spread for each frame. Thus thousands of images are recorded during a $180^{\circ}$ rotation. Right: The PILATUS 6M at the crystallographic beam line X06s at the Swiss Synchrotron Light Source (SLS). 
Crystallization of biomolecules usually takes months to years, whereas X-ray data collection can be done within minutes. In order to solve the molecular structures, the scientist needs the best possible data quality.

With its high quantum efficiency of $100 \%$ at $8 \mathrm{keV}$ and $75 \%$ at $12 \mathrm{keV}$, the exposure time can be minimized. This significantly reduces radiation damage of the crystal. In addition the large detector area with a large number of pixels and small point-spread function (PSF) allows resolving many orders of reflections. The high dynamic range of 1 million (20 bits) per image allows simultaneous detection of weak and strong signals close by each others (Fig. 7).
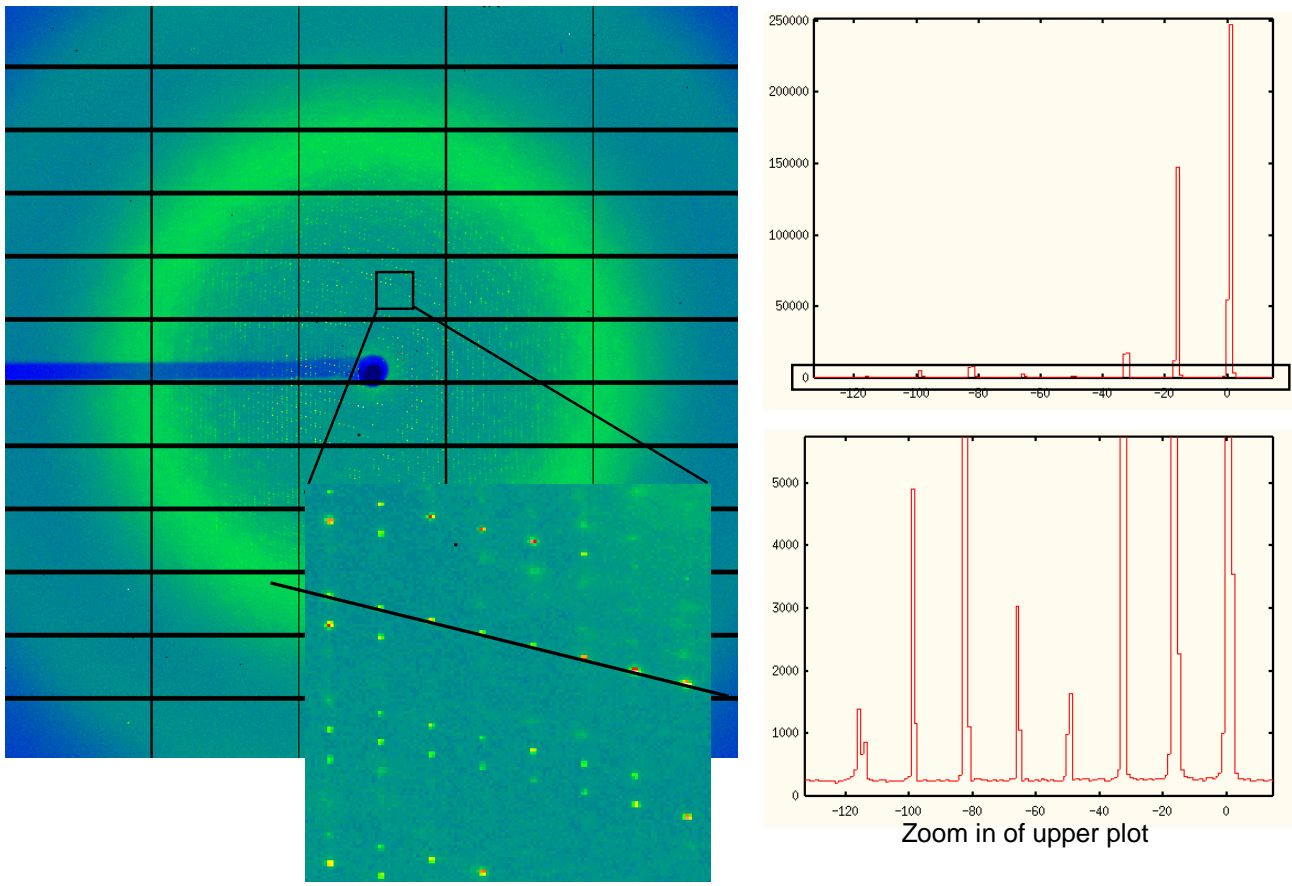

Figure 7: Left: Typical diffraction pattern as produced by a protein crystal. The zoom shows an arbitrary pattern with a large amount of reflections where the intensities vary within several orders of magnitudes (right). (CV. Ramakrishnan, MRC Laboratory of Molecular Biology, Cambridge.

The diffraction pattern of 30s ribosome crystal from the Thermus Thermophilus shows a large amount of reflections. The zoom-in of Fig. 7 shows the impressive regularity of the reflection pattern. The meaning of high dynamic range is revealed by plotting the cross-section through some selected spots. It can be seen that we find spots with 250'000 counts near spots with about 1000 counts which is several orders of magnitudes below. But no matter how intense the peak is, the width is not changed, something that can not be achieved with a CCD.

The PILATUS 6M detector is especially tailored to collect fine phi sliced crystallographic data at high speed in continuous sample rotation mode. In this mode, the crystal is rotated by a fraction of its mosaic spread $\left(0.02-0.2^{\circ}\right)$ during each frame, leading to thousands of images for a $180^{\circ}$ rotation. Such an experiment is very time-consuming with a CCD detector, due to the long readout times. With the PILATUS $6 \mathrm{M}$ detector the crystal can be rotated continuously in the beam without opening and closing the shutter for each frame and waiting for the associated additional spindle movement. Compared to exposure times of approximately $100 \mathrm{~ms}$, the short 
readout time of $3.5 \mathrm{~ms}$ does not compromise the data. And most importantly, unlike CCD detectors, the PILATUS detector does not add any noise to the data during readout. The high frame rate combined with the short readout time reduces the measurement times dramatically.

\subsection{SMALL-ANGLE SCATTERING (SAXS)}

The single-photon counting concept of the PILATUS detectors together with the noiseless readout is an extremely valuable feature, which allows accumulating image frames to result in an almost arbitrarily high dynamic range. This is of interest in small-angle X-ray scattering (SAXS) where the signal range may approach $10^{6}$.

The PILATUS 2M detector was developed in close collaboration with the Coherent Scattering Group [3], Swiss Light Source, Paul Scherrer Institut. The first detector system is installed at the new coherent SAXS beam line X12A.

Applications at this beam line for conventional SAXS experiments range from biological specimens like muscle fibers, bones (Fig. 8), viruses and macromolecular solutions, to problems in soft-condensed-matter physics like ordering of polymers or colloidal suspensions. A growing number of SAXS experiments are carried out in the field of proteins which cannot be crystallized and are thus studied in solutions. These are weakly scattering samples which suffer from radiation damage. The usage of a detector system with high quantum efficiency and a good signal-noise ratio is very important for these studies.
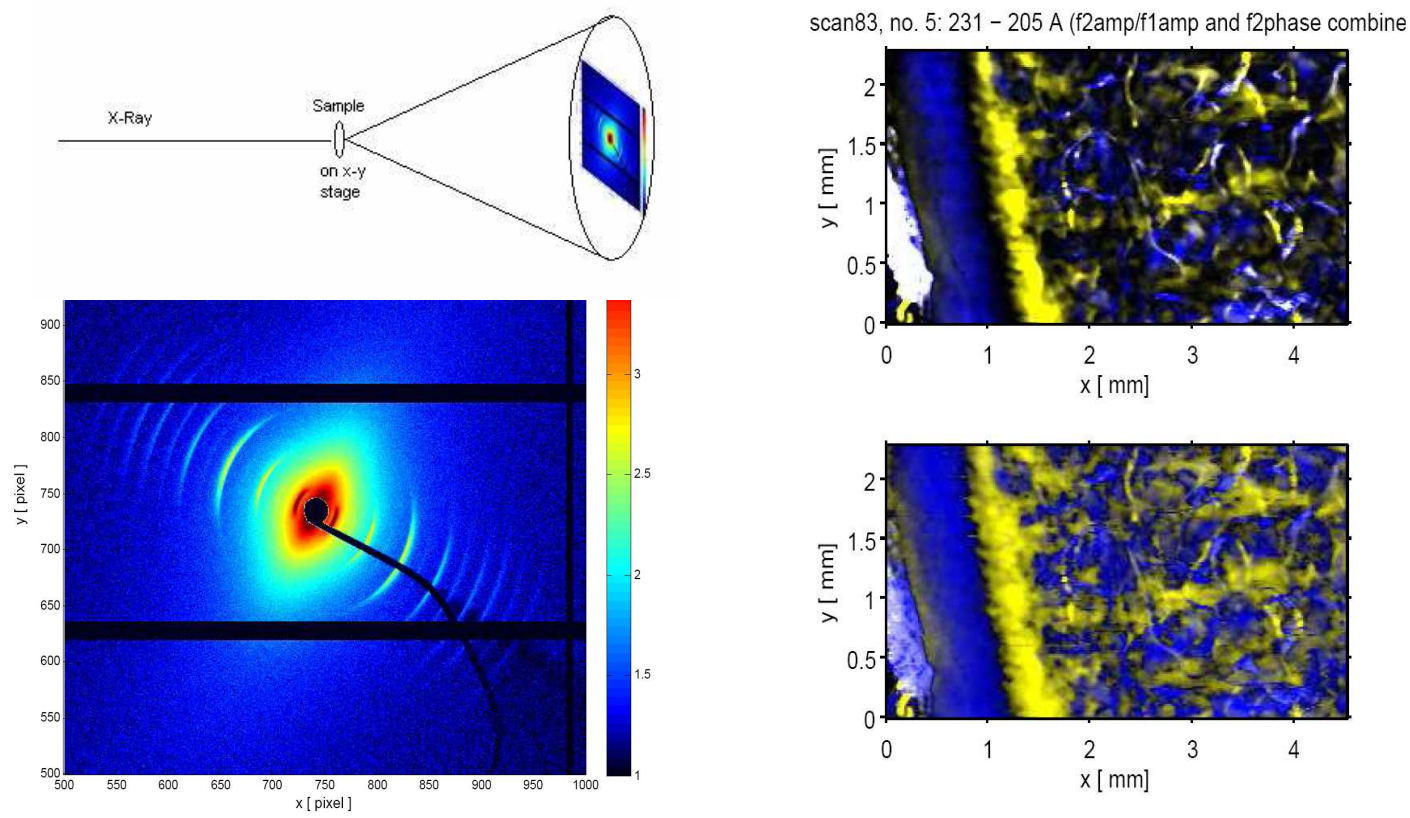

Figure 8: Scan with a focused X-ray beam probing a human piece of the patella bone. Left: A single diffraction image. Right: Integrated intensities of different regions in q-space for each scanning point. (CD.A. Bradley, Centre for Nuclear and Radiation Physics, University of Surrey.

Imaging with structure size contrast down to the $\mathrm{nm}$ regime is a novel application used at the SAXS beam line shown in Fig. 8. The beam was focused to $20 \mu \mathrm{m} \times 6 \mu \mathrm{m}$ making a raster 
scan with step sizes of $15 \mu \mathrm{m}$ on a piece of human patella bone. In total $45^{\prime} 000$ scan points cover a total area of $2.5 \mathrm{~mm} \times 4.5 \mathrm{~mm}$. With this setup the sensitive structure size of $1 \mu \mathrm{m}$ down to $1 \mathrm{~nm}$ was achieved. The integrated intensities of different sections in q-space for each scan point deliver a color coded value to the resulting image. The images in Fig. 8 (right) reveal the preferred collagen fiber orientation in the analyzed bone (yellow).

\subsection{PHASE-CONTRAST IMAGING}

The PSI Coherent Scattering Group [3] in conjunction with the X-Ray Optics and Applications Group [4] have carried out first test experiments to evaluate the performance of the PILATUS $100 \mathrm{~K}$ detector system for medical imaging purposes. Figure 9 shows X-ray images of a test sample (chicken wing) that have been recorded in the conventional absorption mode and in a recently developed novel hard X-ray phase-contrast imaging mode [5].
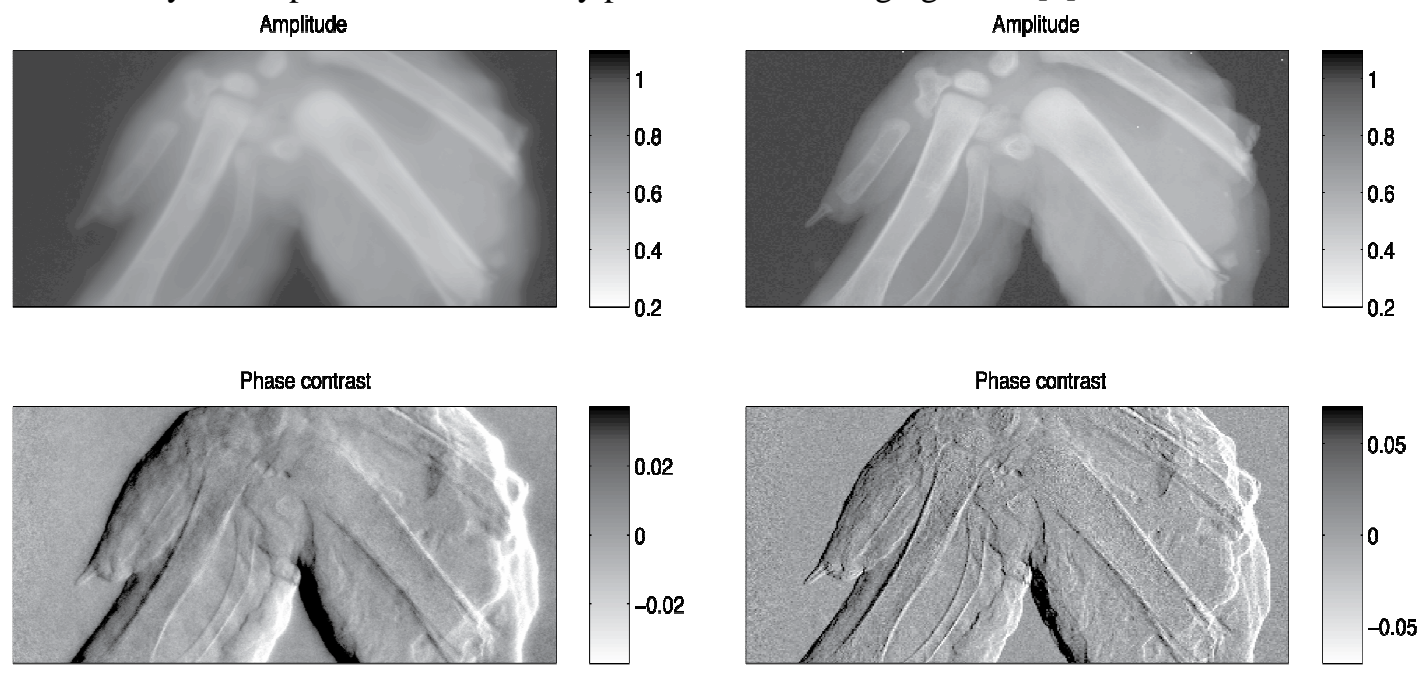

Figure 9: Phase Contrast imaging with a chicken wing. Left: Conventional CCD camera. Right: PILATUS 100K. (Franz Pfeiffer and Oliver Bunk, Paul Scherrer Institut.

A PILATUS 100K (single module detector) was used in competition with a conventional CCD camera. Although the pixel size of PILATUS is almost by a factor ten larger, it delivers much higher contrast. The used CCD consists of a $0.150 \mathrm{~mm}$ thick Cesium Iodide (CsI) scintillation screen with a 1:2.5 demagnifying optical lens system and a cooled charge coupled device (Fingerlakes Instruments, FLI IMG 1001, KODAK chip with 1024 x 1024 pixels, 0.024 x $0.024 \mathrm{~mm}^{2}$ pixel size). Because of the better point spread function the pixel size is not a disadvantage. In contrary it results in a much higher contrast of the image.

The noiseless acquisition and the excellent point-spread-function lead to a very high image quality and demonstrate the good usability of the PILATUS 100K detector system for this kind of application.

\subsection{Quasicrystals}

The Laboratory of Crystallography (ETH) in collaboration with the SLS has studied Bragg reflections of high-quality icosahedral $\mathrm{Al}_{64} \mathrm{Cu}_{23} \mathrm{Fe}_{13}$ using the PILATUS 6M [6]. In theory, 
reflections of icosahedral quasicrystals are densely distributed in reciprocal space. However, not even a tendency towards such a distribution has ever been observed in diffraction experiments of real quasicrystals. In theory the experimentally observable reflection density only depends on the dynamic range of the experiment. Consequently, each increase of the dynamic range leads to more observable Bragg reflections in the case of strictly ordered quasiperiodic structures. The progress in the development of X-ray sources has successively led to higher photon fluxes. Ideally, summation of multiple exposures would lead to an infinite dynamic range and reveal all the weak spots. However, fluorescent background, parasitic scattering and the accumulation of read-out noise have prevented such an observation with common large area detectors.

The high dynamic range in conjunction with the adjustable energy threshold permitting energy discrimination makes the PILATUS an ideal instrument for this purpose.
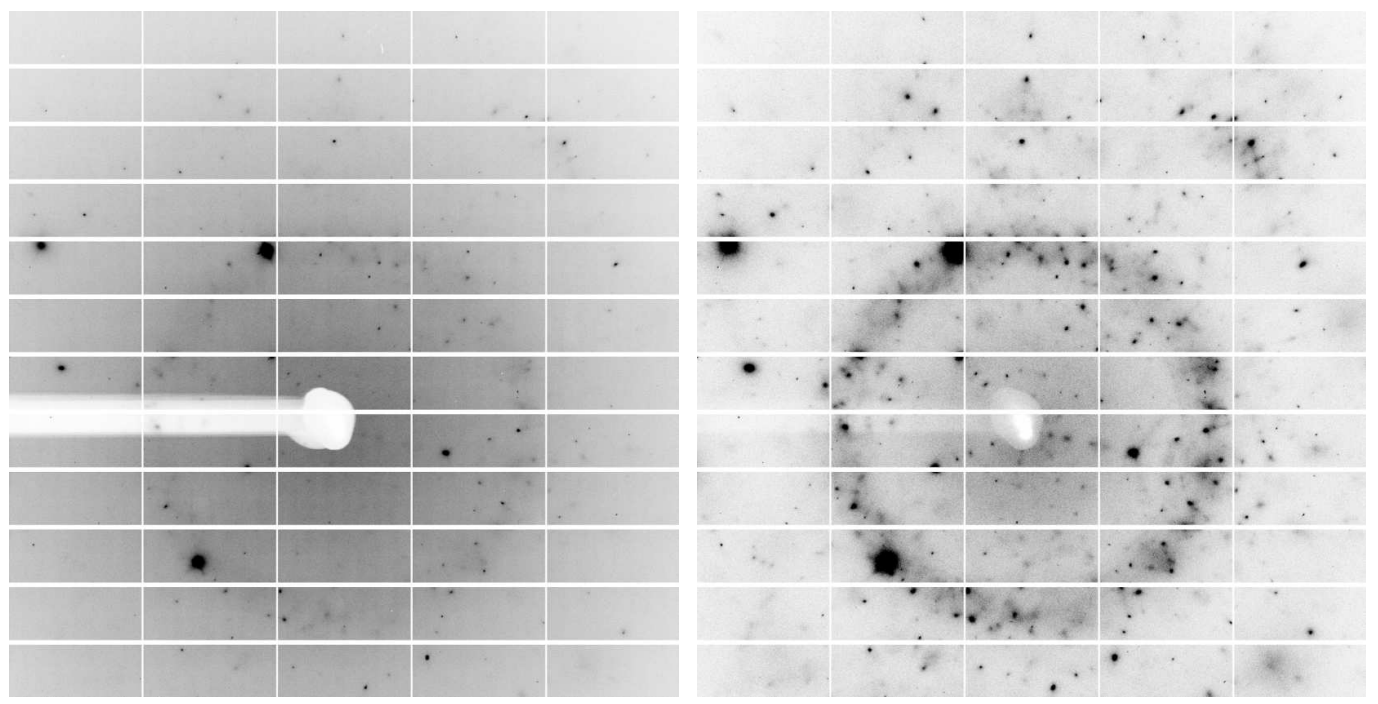

Figure 10: Two single-frame exposures of icosahedral Al64Cu23Fe13 (251 oscillations around $0.1^{\circ}$ each) taken with the energy threshold of the detector set to (left) $8 \mathrm{keV}$ and to (right) $10 \mathrm{keV}$. The intensities are represented by linear grey wedges. () W. Steurer, Laboratory of Crystallography, ETH Zurich.

In a first exposure (Fig. 10, left) the energy threshold of the detector was set such that only radiation with $\mathrm{E} \gamma>8 \mathrm{keV}$ was counted $\left(\mathrm{E}_{\text {source }}=16.02 \mathrm{keV}, \lambda=0.765 \AA\right)$, i.e. scattered photons and fluorescence scattering from $\mathrm{Cu}(7.9 \mathrm{keV})$ was accepted, while fluorescence scattering from Al $(1.4 \mathrm{keV})$ was filtered out. Then, the experiment was repeated with the energy threshold increased to $10 \mathrm{keV}$, completely eliminating fluorescence radiation (Fig. 10, right). A comparison of the figures clearly shows that in the first case the diffraction pattern is dominated by strong Bragg and background scattering, while weak Bragg and diffuse intensities are almost hidden in the background. Most of the background seen on the left is fluorescence scattering from $\mathrm{Cu}$, although the incident beam radiation energy is far from the $\mathrm{Cu}$ absorption edge. In the case of energy integrating detectors such as CCDs or Imaging Plates, the observed background would be even more pronounced because $\mathrm{Fe}$ and $\mathrm{Al}$ fluorescence scattering would be detected as well. In order to check whether increasing exposure time leads to an increasing reflection density as expected for a strictly quasiperiodic crystal, 753 data sets were collected and integrated offline. Surprisingly, a 753 times longer exposure time leads to only a very few additional reflections compared to the many expected for the given experimentally realized 
dynamic range of $10^{9}$. This leads to the conclusion that the local structure of the icosahedral $\mathrm{Al}_{64} \mathrm{Cu}_{23} \mathrm{Fe}_{13}$ quasicrystal differs considerably from the long range ordered average structure.

\section{Summary}

In various applications the PILATUS detector has proven to be a good alternative to current X-ray detector systems. In some aspects it is the superior system. The PILATUS stands out due to features listed below.

- High dynamic range: 1 million (20 bits), which allows to take images with low and very high intensity reflections.

- Short readout times and high frame rates: Read out times of less than $3 \mathrm{~ms}$ and framing rates of up to 200 images per second.

- High local and global counting rates: Each pixel runs at count rates of $>10^{6}$ counts/s.

- Adjustable energy threshold: The energy threshold can be set with an accuracy of $30 \mathrm{eV}$ (measured threshold dispersion and after individual pixel trimming).

- Excellent signal to noise ratio: Inherently, with the single photon counting technique no readout noise is accumulated. A recent measurement confirmed the noise coming from the preamplifier/shaper/comparator stage to be $76 \mathrm{e}$. This gives a discrimination accuracy of $300 \mathrm{eV}$.

- Radiation-tolerant design: On the CMOS design a special radiation-tolerant layout technique (developed for high-energy physics) is used to prevent damage from the incoming X-rays.

- Electronically gateable and external trigger: Exposure times can be varied from $100 \mathrm{~ns}$ to hours and are controlled either internally to an accuracy of $15 \mathrm{~ns}$ or by applying an external gate signal.

- Shutter-less operation: The electronic gating does not require a mechanical shutter; this allows acquiring data in fine phi-slicing mode.

\section{References}

[1] P. Kraft, Characterization of the Readout Chip for the PILATUS 6M Detector, Diploma Thesis, http://pilatus.web.psi.ch/DATA/PDF/Kraft_diploma.pdf.

[2] R. Dinapoli, A Radiation Tolerant Pixel Detector System for the ALICE and LHCb Experiments at CERN, PhD thesis, CERN 2004.

[3] F. Pfeiffer et al, Coherent Scattering Group, PSI, Villigen.

[4] C. David et al, X-Ray Optics and Applications Group, PSI, Villigen.

[5] F. Pfeiffer, T. Weitkamp, O. Bunk, and C. David. Phase retrieval and differential phase-contrast imaging with low-brilliance X-ray source. Nature Physics 2, 258-261 (2006).

[6] Th. Weber et al. Quasicystals. Where are the Reflections? Phys. Rev. Letters, to be published.

[7] P. Kraft et al, http://pilatus.web.psi.ch/DATA/REPORTS/rate scans.html 
[8] L. Rossi, P. Fischer, T. Rohe and N. Wermes, Pixel Detectors: From Fundamentals to Applications, Springer, Berlin (2006).

[9] The Medipix Collaboration: http://medipix.web.cern.ch/MEDIPIX/

[10] N. Boudet, J.-F. Berar, L. Blanquart, P. Breugon, B. Caillot, J.-C. Clemens, I. Koudobine, P. Delpierre, C. Mouget, R. Potheau and I. Valin. Nucl. Instr. and Meth. A 510(2003), p.41-44

[11] B. Mikulec. Nucl. Instr. and Meth. A 510(2003), p.1-23 